\title{
Preparation and Structures of Ternary Copper(II) Complexes of ADP and ATP [1] Models for Enzyme-Metal Ion-Nucleoside Polyphosphate Complexes
}

\author{
William S. Sheldrick \\ Gesellschaft für Biotechnologische Forschung mbH., \\ Mascheroder Weg 1, D-3300 Braunschweig-Stöckheim
}

Z. Naturforsch. 37b, 863-871 (1982); received February 1, 1982

ADP, ATP Copper(II) Ternary Complexes, X-ray

\begin{abstract}
The stable ternary copper(II) complexes of ATP and ADP, $\left[\mathrm{Cu}\left(\mathrm{H}_{2} \mathrm{ATP}\right)(\mathrm{phen})\right]_{2} \cdot 7 \mathrm{H}_{2} \mathrm{O}$ (2) and $\left[\mathrm{Cu}_{4}(\mathrm{HADP})_{2}(\text { bipy })_{4}\left(\mathrm{H}_{2} \mathrm{O}\right)_{2}\left(\mathrm{NO}_{3}\right)_{2}\right] \cdot 2 \mathrm{NO}_{3}(\mathbf{3})$, have been isolated from aqueous solution at respective $\mathrm{pH}$ values of 2.8 and 4.0 . Their structures have been established by single crystal X-ray diffraction. Tridentate coordination of each of the $\mathrm{Cu}$ atoms by one $\alpha$-, one $\beta$ - and one $\gamma$-phosphate O atom of one ATP molecule is observed in 2 . The binding $\mathrm{O}_{\alpha}$ atoms occupy axial positions in a distorted octahedral geometry at $\mathrm{Cu}$ and the $\mathrm{Cu}-\mathrm{O}_{\alpha}$ interactions are weak. The other axial position is occupied by a $\gamma$-phosphate $\mathrm{O}$ atom of the second ATP molecule, leading to a dimeric structure. The basic structure of $\mathbf{3}$ is similar with, in this case, bidentate coordination of each of the central $\mathrm{Cu}$ atoms by one $\alpha$ - and one $\beta$-phosphate $\mathrm{O}$ atom of one ADP molecule. In this case, however, the third terminal $\beta$-phosphate $\mathrm{O}$ atoms each bind a further $\mathrm{Cu}$ atom. All four $\mathrm{Cu}$ atoms in 3 display square pyramidal coordination. The structures of $\mathbf{2}$ and $\mathbf{3}$ are stabilised by intramolecular stacking of adenine and phenanthroline/bipyridyl systems. The significance of these structures as models for enzyme-metal ion-nucleoside polyphosphate complexes is discussed.
\end{abstract}

\section{Introduction}

Enzymes which utilise a nucleoside polyphosphate (e.g. ADP or ATP) as a cofactor or substrate will typically require a specific complex of the nucleotide with a divalent cation for activity. If we consider ATP, in which each of the three phosphate functions is potentially capable of binding the metal ion, then a number of mono-, bi- and tridentate coordination geometries are possible. Furthermore, an enzyme will accept one stereoisomer of such a complex while rejecting others. A detailed knowledge of the molecular geometry of metal-nucleoside polyphosphate complexes is, therefore, essential for an understanding of the modes of enzyme-nucleotide recognition and interaction.

In view of the significant number of $\mathrm{X}$-ray structural characterisations of both binary and ternary metal-nucleotide complexes which have been carried out in the past 7 years $[2,3]$, it is, at first sight, somewhat surprising that these studies have, until very recently, concentrated on nucleoside 5 '-monophosphates. An explanation for this state of affairs must be sought in the inherent instability of metal complexes of ADP and ATP, particularly in the

\footnotetext{
* Full tables of bond lengths and angles and lists of observed and calculated structure factors are available from the author.

0340-5087/82/0700-0863/\$01.00/0
}

solid state. Under physiological conditions the complexed metal ion is commonly $\mathrm{Mg}^{2+}$. Diastereomers of magnesium-nucleoside polyphosphate complexes interconvert with a half-life of the order of only $10^{-5}$ sec. Enzyme function may, however, be studied with other metals, complexes of which may be stable enough to allow separation and characterisation in solution, examples being provided by $\mathrm{Co}\left(\mathrm{NH}_{3}\right)_{n} \mathrm{ADP}$ and $\mathrm{Co}\left(\mathrm{NH}_{3}\right)_{n} \mathrm{HATP}[4,5]$.

Most divalent metal cations catalyse the nonenzymatic transfer of phosphate from nucleoside polyphosphate to various acceptors [6], so that evaporation of solutions of metal salts and ADP or ATP usually yields a mixture of metal phosphates and metal-nucleoside 5'-monophosphate complexes. The introduction of a second chelating ligand such as 2,2'-bipyridyl(bipy) or 1,10-phenanthroline (phen) greatly reduces this dephosphorylation as a result of the formation of very stable ternary complexes between the nucleoside polyphosphate, the metal cation and the chelating ligand. ${ }^{1} \mathrm{H}$ N $Y R$ studies on such complexes in solution have demonstrated that their stability is enhanced by intramolecular stacking interactions between the heterocyclic base and the purine moieties [7]. Recently ternary complexes of $3 \mathrm{~d}$ metals with ATP have also been isolated and characterised in the solid state. Orioli and coworkers have prepared the series 
$\left[\mathrm{M}^{\mathrm{II}}\left(\mathrm{H}_{2} \mathrm{ATP}\right)(\text { bipy) }]_{2} \cdot 4 \mathrm{H}_{2} \mathrm{O}, \mathrm{M}=\mathrm{Mn}^{2+}, \mathrm{Co}^{2+}, \mathrm{Cu}^{2+}\right.$, $\mathrm{Zn}^{2+}[8]$ and have performed an $\mathrm{X}$-ray analysis on the zinc complex (1) [9]. We have synthesised the complex $\left[\mathrm{Cu}\left(\mathrm{H}_{2} \mathrm{ATP}\right)(\text { phen })\right]_{2} \cdot 7 \mathrm{H}_{2} \mathrm{O}$ (2) and have presented a preliminary account of its X-ray structural characterisation [10]. Both 1 and 2, which possess similar structures, display a tridentate coordination of the metal ion. Such complexes are not only of importance as models for the study of the mechanism of the enzymatic phosphate transfer but are also of relevance, on account of their stability towards hydrolysis, as models for the transport of ATP through biological membranes. In this paper we present the preparation and X-ray structural characterisation of a metal-ADP complex, the ternary complex $\left[\mathrm{Cu}_{4}(\mathrm{HADP})_{2}(\text { bipy })_{4}\left(\mathrm{H}_{2} \mathrm{O}\right)_{2}\left(\mathrm{NO}_{3}\right)\right]_{2} \cdot 2 \mathrm{NO}_{3}$ (3). To our knowledge this represents the first isolation of a stable metal-ADP complex in the solid state. We also report a detailed description of the $\mathrm{X}$-ray structure of the ATP complex (2) in comparison with 3 . Under the conditions of preparation, the adenine nitrogen $\mathrm{N}(1)$ is protonated in both 2 and 3 .

\section{Experimental}

Preparation of $\left[\mathrm{Cu}\left(\mathrm{H}_{2} \mathrm{ATP}\right)(\text { phen })\right]_{2} \cdot 7 \mathrm{H}_{2} \mathrm{O}(\mathbf{2})$

$0.10 \mathrm{~g}(0.5 \mathrm{mmol}) \mathrm{Cu}\left(\mathrm{NO}_{3}\right)_{2}$ and $0.10 \mathrm{~g}(0.5 \mathrm{mmol})$ 1,10-phenanthroline in $6 \mathrm{ml}$ of $\mathrm{H}_{2} \mathrm{O}$ were added with stirring to a solution of $0.28 \mathrm{~g}(0.5 \mathrm{mmol}) \mathrm{Na}_{2}$ ATP (Sigma Chemical Co.) in $3 \mathrm{ml} \mathrm{H}_{2} \mathrm{O}$. The $\mathrm{pH}$ value was adjusted to 2.8 and the temperature held at $80{ }^{\circ} \mathrm{C}$ for $30 \mathrm{~min}$. Upon slow cooling of the solution blue-green crystals of $\mathbf{2}$ were precipitated in a quantitative yield. These crystals were filtered off, washed with water and methanol and air dried. The microanalysis, which was carried out by Beller (Göttingen) was in accordance with the presence of either six or seven water molecules of crystallisation. The subsequent X-ray refinement of the structure indicated that the presence of seven water molecules is more probable.

$$
\begin{array}{cccc}
\mathrm{C}_{44} \mathrm{H}_{44} \mathrm{~N}_{14} \mathrm{O}_{26} \mathrm{P}_{6} \mathrm{Cu}_{2} \cdot 7 & \mathrm{H}_{2} \mathrm{O} & \\
\text { Calcd } & \mathrm{C} 32.54 & \mathrm{H} 3.6 & \mathrm{~N} 12.07, \\
\text { Found } & \mathrm{C} 32.8 & \mathrm{H} 3.7 & \mathrm{~N} 12.4 .
\end{array}
$$

\section{Preparation of}

$$
\left[\mathrm{Cu}_{4}(\mathrm{HADP})_{2}(\text { bipy })_{4}\left(\mathrm{H}_{2} \mathrm{O}\right)_{2}\left(\mathrm{NO}_{3}\right)_{2}\right] \cdot 2 \mathrm{NO}_{3}(3)
$$

3 was prepared in a similar manner by adding $0.10 \mathrm{~g}(0.5 \mathrm{mmol}) \mathrm{Cu}\left(\mathrm{NO}_{3}\right)_{2}$ and $0.09 \mathrm{~g}(0.5 \mathrm{mmol})$ $2,2^{\prime}$-bipyridyl in $6 \mathrm{ml}$ of $\mathrm{H}_{2} \mathrm{O}$ to a solution of $0.23 \mathrm{~g}$ (0.5 mmol) NaADP (Sigma Chemical Co.) in $3 \mathrm{ml}$ $\mathrm{H}_{2} \mathrm{O}$ at $80{ }^{\circ} \mathrm{C}$. The $\mathrm{pH}$ value was adjusted to 4.0 . After slow cooling, the solution was allowed to stand in a closed vessel at room temperature. A small quantity of small plate-shaped blue crystals were precipitated over a perios of 3-4 weeks. These were filtered off, washed with water and methanol and air dried. The results of the elementary analysis suggested that water of crystallisation is either absent or that the number of such molecules must be small.

The X-ray analysis provided no evidence for the presence of water of crystallisation.

$$
\begin{array}{cccc}
\mathrm{C}_{60} \mathrm{H}_{62} \mathrm{~N}_{22} \mathrm{O}_{34} \mathrm{P}_{4} \mathrm{Cu}_{4} & & \\
\text { Calcd } & \mathrm{C} 35.79 & \mathrm{H} 3.1 & \mathrm{~N} 15.31, \\
\text { Found } & \mathrm{C} 36.1 & \mathrm{H} 3.4 & \mathrm{~N} 15.2 .
\end{array}
$$

\section{$X$-ray structural analysis of $\mathbf{2}$ and $\mathbf{3}$}

Crystal and refinement data for $\mathbf{2}$ and $\mathbf{3}$ are summarised in Table I. Reflection data were collected in the $\theta-2 \theta$ mode with graphite monochromated $\mathrm{CuK} \alpha$ radiation $(\lambda=1.54178 \AA)$. Empirical absorption corrections based on azimuthal scan data were applied to the intensities. The structures were solved by direct methods and refined by blocked fullmatrix least-squares. The refinement of $\mathbf{2}$ proceeded satisfactorily and anisotropic temperature factors were introduced for the $\mathrm{Cu}, \mathrm{P}$ and $\mathrm{O}$ atoms. Difference syntheses indicated that the presence of 7 water molecules of crystallisation is probable. 4 of these are disordered $-\mathrm{OW}(4)$ to $\mathrm{OW}(7)$. Hydrogen atoms were not included in the refinement. Weights were given by the expression $\mathrm{w}=\mathrm{k}\left[\sigma^{2}\left(\mathrm{~F}_{0}\right)+\mathrm{gF}_{0}^{2}\right]^{-1}$, where $\mathrm{g}$ was fixed at 0.0003 . The final value of $R$ was 0.069 with $R_{\mathrm{w}}=0.067$. Table II lists the final atom coordinates for $\mathbf{2}$, with equivalent isotropic temperature factos $\mathrm{U}_{\text {eq }}=1 / 3 \Sigma_{\mathrm{i}} \Sigma_{\mathrm{j}} \mathrm{U}_{\mathrm{ij}} a_{\mathrm{i}} * a_{\mathrm{j}} * \bar{a}_{\mathrm{i}} \cdot \bar{a}_{\mathrm{j}}$ for the $\mathrm{Cu}, \mathrm{P}$ and $\mathrm{O}$ atoms.

Only very small crystals with limited resolution could be obtained for 3 . The largest of these with dimensions $0.12 \times 0.03 \times 0.14 \mathrm{~mm}$ was used for the data collection. Preliminary crystallographic studies indicated that the solution and refinement of the structure would be difficult if not impossible. A large variation in the mosaic spread of the reflections along the axial directions was observed. Profile widths as measured by $(\omega$-scan were between 1.5 and $2.0^{\circ}$ for the reflections $h 00$ but 3.0 to $3.5^{\circ}$ for reflections $0 k 0$. As a result no reflections significantly greater than background (i.e. with $\mathrm{F}_{0}{ }^{2} \geqslant 2.0 \sigma\left(\mathrm{F}_{0}{ }^{2}\right)$ ) could be observed above $2 \theta=70^{\circ}$ for reflections $h k l$ with $h$ and $l$ small and $k$ large. These reflections display, therefore, a nominal resolution of $1.34 \AA$, which would presumably not have been adequate to allow atom resolution and refinement. However. reflections $h k l$ with $h$ and/or $l$ large and $k$ small were somewhat better resolved and allowed the observation of significant reflections to a $2 \theta$ value of $90^{\circ}$ $($ resolution $=1.09 \AA)$. Of 3296 reflections collected only 1861 showed $\mathrm{F}_{0}{ }^{2} \geqslant 2.0 \sigma\left(\mathrm{F}_{0}{ }^{2}\right)$. Positions for the $\mathrm{Cu}, \mathrm{P}$ and phosphate and ligand water $\mathrm{O}$ atoms were obtained by direct methods and difference syntheses. These atoms were freely refined in the final cycles of refinement and anisotropic temperature factors were introduced for the four $\mathrm{Cu}$ atoms. Conventional 


\begin{tabular}{|c|c|c|}
\hline Compound & $\mathbf{2}$ & 3 \\
\hline Stoichiometry & $\mathrm{C}_{44} \mathrm{H}_{44} \mathrm{~N}_{14} \mathrm{O}_{26} \mathrm{P}_{6} \mathrm{Cu}_{2} \cdot 7 \mathrm{H}_{2} \mathrm{O}$ & {$\left[\mathrm{C}_{60} \mathrm{H}_{62} \mathrm{~N}_{20} \mathrm{O}_{28} \mathrm{P}_{4} \mathrm{Cu}_{4}\right] \cdot 2\left[\mathrm{NO}_{3}\right]$} \\
\hline Space group & $\mathrm{P} 2_{1}$ & $\mathrm{P} 2_{1}$ \\
\hline Crystal size (mm) & $0.36 \times 0.22 \times 0.42$ & $0.12 \times 0.03 \times 0.14$ \\
\hline$a(\AA)$ & $11.807(3)$ & $12.705(4)$ \\
\hline$b(\AA)$ & $24.824(5)$ & $25.279(8)$ \\
\hline$c(\AA)$ & $10.693(2)$ & $12.985(3)$ \\
\hline$\alpha\left({ }^{\circ}\right)$ & 90 & 90 \\
\hline$\beta\left({ }^{\circ}\right)$ & $94.98(3)$ & $95.58(2)$ \\
\hline$\gamma\left({ }^{\circ}\right)$ & 90 & 90 \\
\hline$Z$ & 2 & 2 \\
\hline $\mathrm{M}_{\mathrm{r}}$ & 1624.0 & 2013.4 \\
\hline $\mathrm{D}_{\mathrm{c}}\left(\mathrm{Mgm}^{-3}\right)$ & 1.73 & 1.61 \\
\hline Radiation & $\operatorname{CuK} \alpha$ & $\mathrm{CuK} \alpha$ \\
\hline$\mu\left(\mathrm{cm}^{-1}\right)$ & 30.5 & 25.6 \\
\hline $2 \theta$ range & $\leqslant 120^{\circ}$ & $\leqslant 90^{\circ}$ \\
\hline unique reflections & 4725 & 3296 \\
\hline $\mathrm{F}^{2}$ rejection criterion & $\leqslant 2.0 \sigma$ & $\leqslant 2.0 \sigma$ \\
\hline refinement reflections & 3649 & 1851 \\
\hline$R$ & 0.069 & 0.155 \\
\hline$R_{\mathrm{w}}$ & 0.067 & 0.145 \\
\hline $\mathrm{g}$ & 0.0003 & 0.0005 \\
\hline
\end{tabular}

Table I. Crystal and refinement data.
Table II. Positional parameters and isotropic temperature factors $\left(\AA^{2} \times 10^{3}\right)$ for $2^{\mathrm{a}}$.

\begin{tabular}{|c|c|c|c|c|}
\hline $\begin{array}{l}\text { ATP } \\
\text { molecule } \\
\text { A }\end{array}$ & $x / a$ & $y / b$ & $z / c$ & $\mathrm{U}$ \\
\hline $\mathrm{N}(1)$ & $-0.0571(9)$ & $0.7351(4)$ & $-0.1797(9)$ & $44(3)$ \\
\hline $\mathrm{C}(2)$ & $-0.0360(12)$ & $0.6822(6)$ & $-0.1974(13)$ & $52(4)$ \\
\hline $\mathrm{N}(3)$ & $0.0659(9)$ & $0.6586(4)$ & $-0.1943(10)$ & $46(3)$ \\
\hline $\mathrm{C}(4)$ & $0.1503(10)$ & $0.6949(5)$ & $-0.1668(11)$ & $38(3)$ \\
\hline $\mathrm{C}(5)$ & $0.1409(10)$ & $0.7500(9)$ & $-0.1480(11)$ & $36(3)$ \\
\hline $\mathrm{C}(6)$ & $0.0307(10)$ & $0.7729(5)$ & $-0.1557(11)$ & $33(3)$ \\
\hline $\mathrm{N}(6)$ & $0.0063(8)$ & $0.8240(4)$ & $-0.1449(9)$ & $43(3)$ \\
\hline$N(7)$ & $0.2446(8)$ & $0.7736(4)$ & $-0.1236(9)$ & $42(3)$ \\
\hline $\mathrm{C}(8)$ & $0.3172(10)$ & $0.7340(5)$ & $-0.1332(11)$ & $40(3)$ \\
\hline $\mathrm{N}(9)$ & $0.2651(8)$ & $0.6838(4)$ & $-0.1547(9)$ & $42(3)$ \\
\hline $\mathrm{C}\left(\mathbf{l}^{\prime}\right)$ & $0.3185(10)$ & $0.6292(5)$ & $-0.1612(11)$ & $40(3)$ \\
\hline $\mathrm{O}\left(1^{\prime}\right)$ & $0.4183(7)$ & $0.6302(3)$ & $-0.0772(8)$ & $44(5)$ \\
\hline $\mathrm{C}\left(2^{\prime}\right)$ & $0.3588(13)$ & $0.6201(7)$ & $-0.2919(15)$ & $70(5)$ \\
\hline $\mathrm{O}\left(2^{\prime}\right)$ & $0.3665(10)$ & $0.5609(4)$ & $-0.2966(11)$ & $90(8)$ \\
\hline $\mathrm{C}\left(3^{\prime}\right)$ & $0.4785(12)$ & $0.6428(6)$ & $-0.2750(13)$ & $55(4)$ \\
\hline $\mathrm{O}\left(3^{\prime}\right)$ & $0.5519(10)$ & $0.6204(5)$ & $-0.3621(11)$ & $97(9)$ \\
\hline $\mathrm{C}\left(4^{\prime}\right)$ & $0.5168(12)$ & $0.6246(6)$ & $-0.1456(13)$ & $55(4)$ \\
\hline $\mathrm{C}\left(5^{\prime}\right)$ & $0.6130(13)$ & $0.6579(6)$ & $-0.0751(14)$ & $62(4)$ \\
\hline $\mathrm{O}\left(5^{\prime}\right)$ & $0.5774(7)$ & $0.7123(3)$ & $-0.0639(9)$ & $54(6)$ \\
\hline $\mathrm{P}(1)$ & $0.6626(3)$ & $0.7613(2)$ & $-0.0654(4)$ & $49(2)$ \\
\hline$O(11)$ & $0.5953(7)$ & $0.8105(4)$ & $-0.0341(10)$ & $68(7)$ \\
\hline $\mathrm{O}(12)$ & $0.7203(8)$ & $0.7603(4)$ & $-0.1810(9)$ & $70(7)$ \\
\hline $\mathrm{O}(13)$ & $0.7601(6)$ & $0.7492(3)$ & $0.0434(8)$ & $49(5)$ \\
\hline $\mathrm{P}(2)$ & $0.7586(3)$ & $0.7504(2)$ & $0.1929(4)$ & $51(2)$ \\
\hline $\mathrm{O}(21)$ & $0.6389(8)$ & $0.7570(3)$ & $0.2242(9)$ & $55(6)$ \\
\hline $\mathrm{O}(22)$ & $0.8260(9)$ & $0.7023(5)$ & $0.2428(9)$ & $78(7)$ \\
\hline $\mathrm{O}(23)$ & $0.8306(7)$ & $0.8012(4)$ & $0.2336(9)$ & $59(6)$ \\
\hline $\mathrm{P}(3)$ & $0.7966(3)$ & $0.8659(2)$ & $0.2242(3)$ & $43(2)$ \\
\hline $\mathrm{O}(31)$ & $0.6722(6)$ & $0.8710(3)$ & $0.2330(8)$ & $44(5)$ \\
\hline $\mathrm{O}(32)$ & $0.8271(8)$ & $0.8830(5)$ & $0.0930(8)$ & $73(7)$ \\
\hline $\mathrm{O}(33)$ & $0.8731(7)$ & $0.8905(4)$ & $0.3216(8)$ & $48(5)$ \\
\hline
\end{tabular}

Table II

(continued).

\begin{tabular}{|c|c|c|c|c|}
\hline $\begin{array}{l}\text { ATP } \\
\text { molecule } \\
\text { B }\end{array}$ & $x / a$ & $y / b$ & $z / c$ & $\mathrm{U}$ \\
\hline$N(1)$ & $1.4662(8)$ & $0.9506(4)$ & $0.9329(9)$ & $44(3)$ \\
\hline $\mathrm{C}(2)$ & $1.4504(12)$ & $1.0041(6)$ & $0.9551(13)$ & $53(4)$ \\
\hline $\mathrm{N}(3)$ & $1.3497(9)$ & $1.0263(5)$ & $0.9664(10)$ & $52(3)$ \\
\hline $\mathrm{C}(4)$ & $1.2616(10)$ & $0.9903(5)$ & $0.9506(11)$ & $37(3)$ \\
\hline $\mathrm{C}(5)$ & $1.2749(10)$ & $0.9359(5)$ & $0.9231(11)$ & $33(3)$ \\
\hline $\mathrm{C}(6)$ & $1.3803(10)$ & $0.9138(5)$ & $0.9148(11)$ & $36(3)$ \\
\hline$N(6)$ & $1.4022(9)$ & $0.8627(4)$ & $0.8942(10)$ & $47(3)$ \\
\hline$N(7)$ & $1.1675(8)$ & $0.9127(4)$ & $0.9167(9)$ & $36(2)$ \\
\hline $\mathrm{C}(8)$ & $1.0964(10)$ & $0.9513(5)$ & $0.9390(11)$ & $40(3)$ \\
\hline$N(9)$ & $1.1499(8)$ & $1.0008(4)$ & $0.9598(9)$ & $36(2)$ \\
\hline $\mathrm{C}\left(1^{\prime}\right)$ & $1.0998(10)$ & $1.0518(5)$ & $0.9969(11)$ & $33(3)$ \\
\hline $\mathrm{O}\left(\mathrm{l}^{\prime}\right)$ & $0.9857(7)$ & $1.0400(3)$ & $1.0205(8)$ & $42(5)$ \\
\hline $\mathrm{C}\left(2^{\prime}\right)$ & $1.0962(11)$ & $1.0922(5)$ & $0.8881(12)$ & $42(3)$ \\
\hline $\mathrm{O}\left(2^{\prime}\right)$ & $1.0937(8)$ & $1.1433(3)$ & $0.9450(9)$ & $54(6)$ \\
\hline $\mathrm{C}\left(3^{\prime}\right)$ & $0.9780(11)$ & $1.0784(6)$ & $0.8194(12)$ & $51(4)$ \\
\hline $\mathrm{O}\left(3^{\prime}\right)$ & $0.9329(9)$ & $1.1209(4)$ & $0.7448(10)$ & $75(7)$ \\
\hline $\mathrm{C}\left(4^{\prime}\right)$ & $0.9069(10)$ & $1.0680(5)$ & $0.9314(11)$ & $39(3)$ \\
\hline $\mathrm{C}\left(5^{\prime}\right)$ & $0.8024(11)$ & $1.0329(6)$ & $0.9040(12)$ & $50(4)$ \\
\hline $\mathrm{O}\left(5^{\prime}\right)$ & $0.8376(7)$ & $0.9837(3)$ & $0.8395(8)$ & $44(5)$ \\
\hline $\mathrm{P}(1)$ & $0.7528(3)$ & $0.9343(1)$ & $0.8177(3)$ & $33(2)$ \\
\hline $\mathrm{O}(11)$ & $0.8197(7)$ & $0.8897(3)$ & $0.7755(8)$ & $47(5)$ \\
\hline $\mathrm{O}(12)$ & $0.6861(6)$ & $0.9286(3)$ & $0.9290(7)$ & $37(4)$ \\
\hline $\mathrm{O}(13)$ & $0.6600(6)$ & $0.9555(4)$ & $0.7107(7)$ & $45(5)$ \\
\hline $\mathrm{P}(2)$ & $0.6628(3)$ & $0.9553(2)$ & $0.5618(3)$ & $48(2)$ \\
\hline $\mathrm{O}(21)$ & $0.7802(7)$ & $0.9465(4)$ & $0.5304(9)$ & $56(6)$ \\
\hline $\mathrm{O}(22)$ & $0.6030(9)$ & $1.0040(4)$ & $0.5145(9)$ & $75(7)$ \\
\hline $\mathrm{O}(23)$ & $0.5875(7)$ & $0.9031(4)$ & $0.5203(8)$ & $53(6)$ \\
\hline $\mathrm{P}(3)$ & $0.6168(3)$ & $0.8399(2)$ & $0.5310(3)$ & $48(2)$ \\
\hline $\mathrm{O}(31)$ & $0.7412(7)$ & $0.8311(4)$ & $0.5177(8)$ & $50(5)$ \\
\hline $\mathrm{O}(32)$ & $0.5868(7)$ & $0.8273(4)$ & $0.6648(7)$ & $54(6)$ \\
\hline $\mathrm{O}(33)$ & $0.5388(7)$ & $0.8131(5)$ & $0.4353(8)$ & $79(7)$ \\
\hline
\end{tabular}


Table II (continued).

\begin{tabular}{|c|c|c|c|c|}
\hline $\begin{array}{l}\text { copper } \\
\text { atom } 1\end{array}$ & $x / a$ & $y / b$ & $z / c$ & $\mathrm{U}$ \\
\hline $\mathrm{Cu}(1)$ & $0.5461(1)$ & $0.8216^{\mathrm{b}}$ & $0.2236(2)$ & $41(1)$ \\
\hline $\mathrm{N}(10)$ & $0.4365(8)$ & $0.8823(4)$ & $0.2260(9)$ & $34(2)$ \\
\hline $\mathrm{C}(11)$ & $0.4562(10)$ & $0.9323(5)$ & $0.2481(11)$ & $41(3)$ \\
\hline $\mathrm{C}(12)$ & $0.3673(12)$ & $0.9724(6)$ & $0.2737(13)$ & $54(4)$ \\
\hline $\mathrm{C}(13)$ & $0.2590(12)$ & $0.9555(6)$ & $0.2637(13)$ & $56(4)$ \\
\hline$C(14)$ & $0.2354(12)$ & $0.8983(6)$ & $0.2331(13)$ & $53(4)$ \\
\hline $\mathrm{C}(15)$ & $0.3285(10)$ & $0.8651(5)$ & $0.2225(11)$ & $39(3)$ \\
\hline$C(16)$ & $0.1192(12)$ & $0.8773(6)$ & $0.2281(13)$ & $59(4)$ \\
\hline $\mathrm{C}(17)$ & $0.1047(13)$ & $0.8211(7)$ & $0.2075(14)$ & $68(4)$ \\
\hline $\mathrm{C}(18)$ & $0.1983(12)$ & $0.7864(6)$ & $0.1914(13)$ & $54(4)$ \\
\hline $\mathrm{C}(19)$ & $0.3123(11)$ & $0.8082(5)$ & $0.2002(12)$ & $42(3)$ \\
\hline $\mathrm{N}(20)$ & $0.4039(9)$ & $0.7772(4)$ & $0.1916(10)$ & $43(3)$ \\
\hline $\mathrm{C}(21)$ & $0.3962(14)$ & $0.7246(7)$ & $0.1768(15)$ & $72(5)$ \\
\hline $\mathrm{C}(22)$ & $0.2851(14)$ & $0.6989(7)$ & $0.1609(15)$ & $72(5)$ \\
\hline $\mathrm{C}(23)$ & $0.1886(14)$ & $0.7318(7)$ & $0.1716(15)$ & $76(5)$ \\
\hline $\begin{array}{l}\text { copper } \\
\text { atom } 2\end{array}$ & $x / a$ & $y / b$ & $z / c$ & $\mathrm{U}$ \\
\hline $\mathrm{Cu}(2)$ & $0.8692(2)$ & $0.8790(1)$ & $0.5321(2)$ & $57(1)$ \\
\hline $\mathbf{N}(10)$ & $0.9765(9)$ & $0.8141(5)$ & $0.5314(10)$ & $54(3)$ \\
\hline $\mathrm{C}(11)$ & $0.9442(12)$ & $0.7629(6)$ & $0.5036(14)$ & $58(4)$ \\
\hline $\mathrm{C}(12)$ & $1.0266(14)$ & $0.7236(7)$ & $0.4952(15)$ & $71(5)$ \\
\hline $\mathrm{C}(13)$ & $1.1420(15)$ & $0.7404(8)$ & $0.5102(16)$ & $82(5)$ \\
\hline $\mathrm{C}(14)$ & $1.1763(12)$ & $0.7906(6)$ & $0.5319(13)$ & $52(4)$ \\
\hline $\mathrm{C}(15)$ & $1.0880(11)$ & $0.8316(6)$ & $0.5475(12)$ & $47(3)$ \\
\hline $\mathrm{C}(16)$ & $1.2917(17)$ & $0.8124(9)$ & $0.5546(18)$ & $101(6)$ \\
\hline $\mathrm{C}(17)$ & $1.3129(13)$ & $0.8602(7)$ & $0.5725(15)$ & $69(5)$ \\
\hline $\mathrm{C}(18)$ & $1.2207(14)$ & $0.9042(7)$ & $0.5791(15)$ & $66(4)$ \\
\hline $\mathrm{C}(19)$ & $1.1106(11)$ & $0.8824(6)$ & $0.5644(12)$ & $47(3)$ \\
\hline $\mathrm{N}(20)$ & $1.0165(9)$ & $0.9175(5)$ & $0.5686(10)$ & $53(3)$ \\
\hline $\mathrm{C}(21)$ & $1.0344(13)$ & $0.9692(7)$ & $0.5840(14)$ & $63(4)$ \\
\hline $\mathrm{C}(22)$ & $1.1483(16)$ & $0.9898(8)$ & $0.5957(17)$ & $87(6)$ \\
\hline $\mathrm{C}(23)$ & $1.2360(14)$ & $0.9555(7)$ & $0.5996(15)$ & $73(5)$ \\
\hline$O W(1)^{c}$ & $0.5233(15)$ & $0.6984(8)$ & $0.4511(16)$ & $174(7)$ \\
\hline $\mathrm{OW}(2)$ & $0.7905(15)$ & $0.5873(8)$ & $0.6840(17)$ & $171(7)$ \\
\hline OW(3) & $0.7466(19)$ & $0.6296(10)$ & $0.4029(21)$ & $240(10)$ \\
\hline
\end{tabular}

a The $\mathrm{Cu}, \mathrm{P}$ and ATP $\mathrm{O}$ atoms were refined anisotropically; equivalent isotropic temperature factors are listed for these atoms;

$\mathrm{b}$ the $y$ coordinate of $\mathrm{Cu}(\mathbf{l})$ was not refined in order to fix the origin;

c the water oxygens $\mathrm{OW}(4)-\mathrm{OW}(7)$ are disordered.

least-squares refinement of the adenosine, bipyridyl and nitrate ligands and anions was not possible. Difference syntheses allowed the location of probable atom positions and these were refined in a constrained manner. For the adenosine moieties standard bond lengths were employed [11]. The bipyridyl rings were refined as regular hexagons with $\mathrm{d}(\mathrm{C}-\mathrm{C})$ and $\mathrm{d}(\mathrm{C}-\mathrm{N})=1.395 \AA$. The nitrate ligands and anions were refined to be planar with $\mathrm{d}(\mathrm{N}-\mathrm{O})=$ $1.21 \AA$. With the exception of the $\mathrm{Cu}$ and $\mathrm{P}$ atoms group isotropic temperature factors were employed (Table III). Difference syntheses did not indicate the presence of water molecules of crystallisation. However because of the poor quality of the data and the possibility of disorder, no definite conclusion may be drawn.

To allow for the differences in the mosaic spread, the intensity data were rescaled anisotropically using the method of Shakked and Rabinovich [12]

$$
\mathrm{F}_{0}(\text { scaled })=\mathrm{F}_{0} / \mathrm{T}_{\mathrm{H}}
$$

where $\mathrm{T}_{\mathrm{H}}=\mathrm{i}, \mathrm{j} \stackrel{3}{\Sigma}_{1} h_{\mathrm{i}} h_{\mathrm{j}} a_{\mathrm{i}} * a_{\mathrm{j}} * \mathrm{~T}_{\mathrm{ij} / \mathrm{i}, \mathrm{j}} \stackrel{3}{\Sigma_{1}}\left(h_{\mathrm{i}} a_{\mathrm{i}}^{*}\right) \cdot\left(h_{\mathrm{j}} a_{\mathrm{j}} *\right)$.

The components of the svmmetric scaling tensor were refined by least-squares to the following values: $\mathrm{T}_{11}=1.13(1), \mathrm{T}_{22}=0.52(1), \mathrm{T}_{33}=1.17(1), \mathrm{T}_{13}=$ $0.12(1)$. Without this rescaling $R$ was 0.193 . Rescaling led to the final $R$ factor of 0.155 with $R_{\mathrm{w}}=$ 0.144 . These values are satisfactory in view of the very limited quality of the data. Table III lists the final atom coordinates for $\mathbf{3}$, with equivalent isotropic temperature factors for the $\mathrm{Cu}$ atoms.

Selected bond lengths and angles at the copper atoms and in the triphosphate chains of $\mathbf{2}$ are listed in Table IV. These quantities in 3 were not determined to sufficient precision to warrant their listing. Crystallographic calculations were performed with SHELX (G. M. Sheldrick) and locally developed programs. Figs. 1-4 were drawn with RSPLOT (W. S. Sheldrick).

Table III. Positional parameters and isotropic temperature factors $\left(\AA \times 10^{3}\right)$ for $3^{\mathrm{a}}$.

\begin{tabular}{|c|c|c|c|c|}
\hline $\begin{array}{l}\text { ADP } \\
\text { mole- } \\
\text { cule A }\end{array}$ & $x / a$ & $y / b$ & $z / c$ & $\mathrm{U}$ \\
\hline$N(1)$ & $-0.0010(41)$ & $0.1022(26)$ & $0.4407(39)$ & $65(7)$ \\
\hline $\mathrm{C}(2)$ & $0.0032(47)$ & $0.0513(26)$ & $0.4161(48)$ & $65(7)$ \\
\hline $\mathrm{N}(3)$ & $0.0793(43)$ & $0.0421(23)$ & $0.3557(40)$ & $65(7)$ \\
\hline $\mathrm{C}(4)$ & $0.1424(48)$ & $0.0800(26)$ & $0.3220(46)$ & $65(7)$ \\
\hline $\mathrm{C}(5)$ & $0.1168(48)$ & $0.1323(26)$ & $0.3315(45)$ & $65(7)$ \\
\hline $\mathrm{C}(6)$ & $0.0358(48)$ & $0.1451(23)$ & $0.3930(50)$ & $65(7)$ \\
\hline $\mathrm{N}(6)$ & $0.0180(41)$ & $0.1974(23)$ & $0.3941(40)$ & $65(7)$ \\
\hline$N(7)$ & $0.1769(39)$ & $0.1522(23)$ & $0.2563(39)$ & $65(7)$ \\
\hline $\mathrm{C}(8)$ & $0.2238(51)$ & $0.1119(23)$ & $0.2184(49)$ & $65(7)$ \\
\hline $\mathrm{N}(9)$ & $0.2053(42)$ & $0.0612(23)$ & $0.2499(41)$ & $65(7)$ \\
\hline$C\left(1^{\prime}\right)$ & $0.2378(50)$ & $0.0063(25)$ & $0.2289(49)$ & $132(11)$ \\
\hline$O\left(1^{\prime}\right)$ & $0.3467(49)$ & $-0.0039(26)$ & $0.2258(46)$ & $132(11)$ \\
\hline $\mathrm{C}\left(2^{\prime}\right)$ & $0.2043(63)$ & $0.0087(25)$ & $0.1133(47)$ & $132(11)$ \\
\hline $\mathrm{O}\left(2^{\prime}\right)$ & $0.1806(43)$ & $-0.0451(25)$ & $0.1276(40)$ & $132(11)$ \\
\hline $\mathrm{C}\left(3^{\prime}\right)$ & $0.2951(65)$ & $-0.0135(37)$ & $0.0570(53)$ & $132(11)$ \\
\hline $\mathrm{O}\left(3^{\prime}\right)$ & $0.3019(46)$ & $-0.0337(25)$ & $-0.0433(46)$ & $132(11)$ \\
\hline $\mathrm{C}\left(4^{\prime}\right)$ & $0.3852(60)$ & $-0.0306(32)$ & $0.1368(57)$ & $132(11)$ \\
\hline $\mathrm{C}\left(5^{\prime}\right)$ & $0.4694(73)$ & $0.0049(26)$ & $0.0994(72)$ & $132(11)$ \\
\hline $\mathrm{O}\left(5^{\prime}\right)$ & $0.4790(39)$ & $0.0614(25)$ & $0.1026(39)$ & $100(10)$ \\
\hline $\mathrm{P}(1)$ & $0.5511(17)$ & $0.0959(11)$ & $0.1873(16)$ & $75(8)$ \\
\hline $\mathrm{O}(11)$ & $0.4776(33)$ & $0.1150(13)$ & $0.2653(32)$ & $100(10)$ \\
\hline $\mathrm{O}(12)$ & $0.6669(39)$ & $0.0702(22)$ & $0.2136(37)$ & $100(10)$ \\
\hline$O(13)$ & $0.5721(38)$ & $0.1503(24)$ & $0.1160(39)$ & $100(10)$ \\
\hline $\mathrm{P}(2)$ & $0.5447(13)$ & $0.2105(9)$ & $0.1297(13)$ & $34(5)$ \\
\hline $\mathrm{O}(21)$ & $0.5242(29)$ & $0.2128(18)$ & $0.2426(28)$ & $48(7)$ \\
\hline $\mathrm{O}(22)$ & $0.4422(29)$ & $0.2129(18)$ & $0.0445(28)$ & $48(7)$ \\
\hline $\mathrm{O}(23)$ & $0.6455(29)$ & $0.2357(18)$ & $0.1024(27)$ & $48(7)$ \\
\hline
\end{tabular}


Table III (continued).

\begin{tabular}{|c|c|c|c|c|}
\hline $\begin{array}{l}\text { ADP } \\
\text { mole- } \\
\text { cule B }\end{array}$ & $x / a$ & $y / b$ & $z / c$ & $\mathrm{U}$ \\
\hline $\mathrm{N}(1)$ & $1.2727(34)$ & $0.3318(20)$ & $0.1208(34)$ & $35(6)$ \\
\hline $\mathrm{C}(2)$ & $1.2382(41)$ & $0.3815(21)$ & $0.1218(39)$ & $35(6)$ \\
\hline $\mathrm{N}(3)$ & $1.1802(33)$ & $0.4055(18)$ & $0.1868(33)$ & $35(6)$ \\
\hline$C(4)$ & $1.1372(39)$ & $0.3696(21)$ & $0.2472(38)$ & $35(6)$ \\
\hline $\mathrm{C}(5)$ & $1.1479(38)$ & $0.3161(21)$ & $0.2355(39)$ & $35(6)$ \\
\hline $\mathrm{C}(6)$ & $1.2304(39)$ & $0.2964(19)$ & $0.1826(40)$ & $35(6)$ \\
\hline $\mathrm{N}(6)$ & $1.2439(33)$ & $0.2446(19)$ & $0.1661(32)$ & $35(6)$ \\
\hline $\mathrm{N}(7)$ & $1.0866(34)$ & $0.2891(18)$ & $0.3016(34)$ & $35(6)$ \\
\hline $\mathrm{C}(8)$ & $1.0466(42)$ & $0.3259(20)$ & $0.3555(40)$ & $35(6)$ \\
\hline $\mathrm{N}(9)$ & $1.0645(33)$ & $0.3753(21)$ & $0.3175(33)$ & $35(6)$ \\
\hline $\mathrm{C}\left(\mathrm{l}^{\prime}\right)$ & $1.0372(44)$ & $0.4284(21)$ & $0.3544(34)$ & $65(7)$ \\
\hline $\mathrm{O}\left(1^{\prime}\right)$ & $0.9885(32)$ & $0.4225(19)$ & $0.4470(30)$ & $65(7)$ \\
\hline $\mathrm{C}\left(2^{\prime}\right)$ & $0.9654(39)$ & $0.4562(21)$ & $0.2710(42)$ & $65(7)$ \\
\hline $\mathrm{O}\left(2^{\prime}\right)$ & $0.9710(33)$ & $0.5098(22)$ & $0.3019(32)$ & $65(7)$ \\
\hline $\mathrm{C}\left(3^{\prime}\right)$ & $0.8566(38)$ & $0.4474(25)$ & $0.3094(39)$ & $65(7)$ \\
\hline $\mathrm{O}\left(3^{\prime}\right)$ & $0.7679(33)$ & $0.4792(18)$ & $0.2784(32)$ & $65(7)$ \\
\hline $\mathrm{C}\left(4^{\prime}\right)$ & $0.8760(33)$ & $0.4342(27)$ & $0.4248(39)$ & $65(7)$ \\
\hline $\mathrm{C}\left(5^{\prime}\right)$ & $0.8055(48)$ & $0.3993(19)$ & $0.4832(47)$ & $65(7)$ \\
\hline $\mathrm{O}\left(5^{\prime}\right)$ & $0.8186(29)$ & $0.3458(19)$ & $0.4511(29)$ & $46(7)$ \\
\hline $\mathrm{P}(1)$ & $0.7232(12)$ & $0.3203(8)$ & $0.3663(12)$ & $27(5)$ \\
\hline $\mathrm{O}(11)$ & $0.7754(29)$ & $0.3024(17)$ & $0.2766(29)$ & $46(7)$ \\
\hline $\mathrm{O}(12)$ & $0.6397(29)$ & $0.3628(14)$ & $0.3427(28)$ & $46(7)$ \\
\hline $\mathrm{O}(13)$ & $0.6943(26)$ & $0.2678(10)$ & $0.4218(26)$ & $46(7)$ \\
\hline $\mathrm{P}(2)$ & $0.7185(15)$ & $0.2072(10)$ & $0.4201(15)$ & $55(6)$ \\
\hline $\mathrm{O}(21)$ & $0.7468(27)$ & $0.1914(16)$ & $0.3173(27)$ & $39(7)$ \\
\hline $\mathrm{O}(22)$ & $0.8154(28)$ & $0.2016(17)$ & $0.4962(27)$ & $39(7)$ \\
\hline $\mathrm{O}(23)$ & $0.6211(27)$ & $0.1837(17)$ & $0.4481(26)$ & $39(7)$ \\
\hline
\end{tabular}

\section{Copper}

atom 1

\begin{tabular}{llllr}
\hline $\mathrm{Cu}(1)$ & $0.4383(7)$ & $0.2572^{\mathrm{b}}$ & $-0.0787(7)$ & $79(9)$ \\
$\mathrm{N}(10)$ & $0.4717(28)$ & $0.2961(13)-0.2070(32)$ & $38(6)$ \\
$\mathrm{C}(11)$ & $0.4730(28)$ & $0.3500(13)-0.2300(32)$ & $38(6)$ \\
$\mathrm{C}(12)$ & $0.5074(28)$ & $0.3670(13)-0.3235(32)$ & $38(6)$ \\
$\mathrm{C}(13)$ & $0.5403(28)$ & $0.3302(13)-0.3936(32)$ & $38(6)$ \\
$\mathrm{C}(14)$ & $0.5390(28)$ & $0.2763(13)-0.3705(32)$ & $38(6)$ \\
$\mathrm{C}(15)$ & $0.5047(28)$ & $0.2593(13)-0.2771(32)$ & $38(6)$ \\
$\mathrm{C}(16)$ & $0.5027(30)$ & $0.2003(13)-0.2559(33)$ & $38(6)$ \\
$\mathrm{N}(17)$ & $0.4620(30)$ & $0.1860(13)-0.1639(33)$ & $38(6)$ \\
$\mathrm{C}(18)$ & $0.4523(30)$ & $0.1327(13)-0.1386(33)$ & $38(6)$ \\
$\mathrm{C}(19)$ & $0.4833(30)$ & $0.0937(13)-0.2053(33)$ & $38(6)$ \\
$\mathrm{C}(20)$ & $0.5240(30)$ & $0.1080(13)-0.2973(33)$ & $38(6)$ \\
$\mathrm{C}(21)$ & $0.5337(30)$ & $0.1613(13)-0.3226(33)$ & $38(6)$ \\
$\mathrm{OW}(1)$ & $0.2611(34)$ & $0.2524(23)-0.1096(33)$ & $86(11)$ \\
$\mathrm{O}(1)$ & $0.4473(29)$ & $0.3236(20)-0.0004(29)$ & $86(11)$ \\
$\mathrm{N}(2)$ & $0.5110(28)$ & $0.3433(20)$ & $0.0663(28)$ & $86(11)$ \\
$\mathrm{O}(3)$ & $0.4902(40)$ & $0.3421(26)$ & $0.1566(28)$ & $152(19)$ \\
$\mathrm{O}(4)$ & $0.6032(29)$ & $0.3495(26)$ & $0.0486(39)$ & $152(19)$ \\
\hline
\end{tabular}

Copper

\begin{tabular}{lllll} 
atom 2 & $x / a$ & $y / b$ & $z / c$ & $\mathrm{U}$ \\
\hline $\mathrm{Cu}(2)$ & $0.4619(7)$ & $0.1804(5)$ & $0.3413(7)$ & $61(9)$ \\
$\mathrm{N}(10)$ & $0.3989(35)$ & $0.2444(15)$ & $0.4077(31)$ & $54(6)$ \\
$\mathrm{C}(11)$ & $0.4114(35)$ & $0.2961(15)$ & $0.3734(31)$ & $54(6)$ \\
$\mathrm{C}(12)$ & $0.3481(35)$ & $0.3365(15)$ & $0.4069(31)$ & $54(6)$ \\
$\mathrm{C}(13)$ & $0.2723(35)$ & $0.3252(15)$ & $0.4746(31)$ & $54(6)$ \\
$\mathrm{C}(14)$ & $0.2599(35)$ & $0.2735(15)$ & $0.5089(31)$ & $54(6)$ \\
$\mathrm{C}(15)$ & $0.3232(35)$ & $0.2331(15)$ & $0.4754(31)$ & $54(6)$ \\
$\mathrm{C}(16)$ & $0.3877(26)$ & $0.1435(20)$ & $0.4478(24)$ & $54(6)$ \\
\hline
\end{tabular}

Table III (continued).

\begin{tabular}{lllll}
\hline & $x / a$ & $y / b$ & $z / c$ & $\mathrm{U}$ \\
\hline $\mathrm{N}(17)$ & $0.3825(26)$ & $0.0893(20)$ & $0.4669(24)$ & $54(6)$ \\
$\mathrm{C}(18)$ & $0.3128(26)$ & $0.0699(20)$ & $0.5345(24)$ & $54(6)$ \\
$\mathrm{C}(19)$ & $0.2481(26)$ & $0.1048(20)$ & $0.5829(24)$ & $54(6)$ \\
$\mathrm{C}(20)$ & $0.2532(26)$ & $0.1590(20)$ & $0.5637(24)$ & $54(6)$ \\
$\mathrm{C}(21)$ & $0.3230(26)$ & $0.1783(20)$ & $0.4962(24)$ & $54(6)$ \\
\hline $\mathrm{Copper}$ & & & & \\
atom 3 & $x / a$ & $y / b$ & $z / c$ & $\mathrm{U}$ \\
& & & & \\
$\mathrm{Cu}(3)$ & $0.7989(7)$ & $0.2403(6)$ & $0.2036(7)$ & $86(10)$ \\
$\mathrm{N}(10)$ & $0.8804(31)$ & $0.1734(14)$ & $0.1507(31)$ & $40(5)$ \\
$\mathrm{C}(11)$ & $0.8689(31)$ & $0.1210(14)$ & $0.1809(31)$ & $40(5)$ \\
$\mathrm{C}(12)$ & $0.9133(31)$ & $0.0803(14)$ & $0.1267(31)$ & $40(5)$ \\
$\mathrm{C}(13)$ & $0.9692(31)$ & $0.0931(14)$ & $0.0424(31)$ & $40(5)$ \\
$\mathrm{C}(14)$ & $0.9808(31)$ & $0.1446(14)$ & $0.0122(31)$ & $40(5)$ \\
$\mathrm{C}(15)$ & $0.9364(31)$ & $0.1852(14)$ & $0.0663(31)$ & $40(5)$ \\
$\mathrm{C}(16)$ & $0.9544(32)$ & $0.2485(13)$ & $0.0460(31)$ & $40(5)$ \\
$\mathrm{C}(17)$ & $0.8898(32)$ & $0.2793(13)$ & $0.1029(31)$ & $40(5)$ \\
$\mathrm{C}(18)$ & $0.8892(32)$ & $0.3342(13)$ & $0.0922(31)$ & $40(5)$ \\
$\mathrm{C}(19)$ & $0.9533(32)$ & $0.3583(13)$ & $0.0245(31)$ & $40(5)$ \\
$\mathrm{C}(20)$ & $1.0180(32)$ & $0.3275(13)$ & $-0.0324(31)$ & $40(5)$ \\
$\mathrm{C}(21)$ & $1.0186(32)$ & $0.2726(13)$ & $-0.0217(31)$ & $40(5)$ \\
& & & &
\end{tabular}

Copper

\begin{tabular}{lllll} 
atom 4 & $x / a$ & $y / b$ & $z / c$ & $\mathrm{U}$ \\
\hline $\mathrm{Cu}(4)$ & $0.8230(7)$ & $0.1611(5)$ & $0.6304(7)$ & $61(9)$ \\
$\mathrm{N}(10)$ & $0.7978(27)$ & $0.1382(13)$ & $0.7632(30)$ & $30(5)$ \\
$\mathrm{C}(11)$ & $0.7998(27)$ & $0.0838(13)$ & $0.7815(30)$ & $30(5)$ \\
$\mathrm{C}(12)$ & $0.7610(27)$ & $0.0635(13)$ & $0.8703(30)$ & $30(5)$ \\
$\mathrm{C}(13)$ & $0.7202(27)$ & $0.0977(13)$ & $0.9408(30)$ & $30(5)$ \\
$\mathrm{C}(14)$ & $0.7182(27)$ & $0.1521(13)$ & $0.9226(30)$ & $30(5)$ \\
$\mathrm{C}(15)$ & $0.7570(27)$ & $0.1723(13)$ & $0.8338(30)$ & $30(5)$ \\
$\mathrm{C}(16)$ & $0.7691(28)$ & $0.2175(14)$ & $0.8116(28)$ & $30(5)$ \\
$\mathrm{N}(17)$ & $0.8011(28)$ & $0.2222(14)$ & $0.7123(28)$ & $30(5)$ \\
$\mathrm{C}(18)$ & $0.8101(28)$ & $0.2721(14)$ & $0.6682(28)$ & $30(5)$ \\
$\mathrm{C}(19)$ & $0.7871(28)$ & $0.3173(14)$ & $0.7235(28)$ & $30(5)$ \\
$\mathrm{C}(20)$ & $0.7551(28)$ & $0.3126(14)$ & $0.8228(28)$ & $30(5)$ \\
$\mathrm{C}(21)$ & $0.7461(28)$ & $0.2626(14)$ & $0.8669(28)$ & $30(5)$ \\
$\mathrm{OW}(2)$ & $0.9975(24)$ & $0.1668(17)$ & $0.6480(24)$ & $27(7)$ \\
$\mathrm{O}(5)$ & $0.8204(23)$ & $0.0852(14)$ & $0.5477(25)$ & $27(7)$ \\
$\mathrm{N}(6)$ & $0.7514(24)$ & $0.0589(13)$ & $0.4982(25)$ & $27(7)$ \\
$\mathrm{O}(7)$ & $0.6635(27)$ & $0.0781(19)$ & $0.4766(40)$ & $136(17)$ \\
$\mathrm{O}(8)$ & $0.7558(37)$ & $0.0107(13)$ & $0.5099(42)$ & $136(17)$
\end{tabular}

Nitrate

\begin{tabular}{lllll} 
anions & $x / a$ & \multicolumn{1}{l}{$y / b$} & \multicolumn{1}{l}{$z / c$} & $\mathrm{U}$ \\
\hline $\mathrm{N}$ & $0.1731(43)$ & $0.1153(18)$ & $-0.1215(34)$ & $135(30)$ \\
$\mathrm{O}$ & $0.2327(48)$ & $0.1270(25)$ & $-0.0454(37)$ & $187(19)$ \\
$\mathrm{O}$ & $0.1485(49)$ & $0.1490(22)$ & $-0.1868(42)$ & $187(19)$ \\
$\mathrm{O}$ & $0.1591(52)$ & $0.0686(18)$ & $-0.1429(47)$ & $187(19)$ \\
$\mathrm{N}$ & $0.0893(39)$ & $0.3007(19)$ & $0.7030(41)$ & $135(29)$ \\
$\mathrm{O}$ & $0.0335(38)$ & $0.3168(22)$ & $0.6279(36)$ & $187(15)$ \\
$\mathrm{O}$ & $0.0706(41)$ & $0.2587(18)$ & $0.7436(40)$ & $187(15)$ \\
$\mathrm{O}$ & $0.1708(36)$ & $0.3243(21)$ & $0.7315(41)$ & $187(15)$
\end{tabular}

a Equivalent isotropic temperature factors are given for the $\mathrm{Cu}$ atoms, which were refined anisotropically; $\mathrm{b}$ the $y$ coordinate of $\mathrm{Cu}(1)$ was not refined in order to fix the origin. 
a) Bond lengths $(\AA)$

\begin{tabular}{|c|c|c|c|}
\hline $\mathrm{Cu}(1)-\mathrm{O}(11 \mathrm{~A})$ & $2.878(9)$ & $\mathrm{Cu}(1)-\mathrm{O}(21 \mathrm{~A})$ & $1.942(9)$ \\
\hline $\mathrm{Cu}(1)-\mathrm{O}(31 \mathrm{~A})$ & $1.925(8)$ & $\mathrm{Cu}(1)-\mathrm{O}(33 \mathrm{~B})$ & $2.284(8)$ \\
\hline $\mathrm{Cu}(1)-\mathrm{N}(10 \mathrm{~A})$ & $1.989(10)$ & $\mathrm{Cu}(1)-\mathrm{N}(20 \mathrm{~A})$ & $2.013(10)$ \\
\hline $\mathrm{P}(1 \mathrm{~A})-\mathrm{O}\left(5^{\prime} \mathrm{A}\right)$ & $1.578(9)$ & $\mathrm{P}(1 \mathrm{~A})-\mathrm{O}(11 \mathrm{~A})$ & $1.512(10)$ \\
\hline $\mathrm{P}(1 \mathrm{~A})-\mathrm{O}(12 \mathrm{~A})$ & $1.462(11)$ & $\mathrm{P}(1 \mathrm{~A})-\mathrm{O}(13 \mathrm{~A})$ & $1.593(9)$ \\
\hline $\mathrm{P}(2 \mathrm{~A})-\mathrm{O}(13 \mathrm{~A})$ & $1.600(10)$ & $\mathrm{P}(2 \mathrm{~A})-\mathrm{O}(21 \mathrm{~A})$ & $1.488(10)$ \\
\hline $\mathrm{P}(2 \mathrm{~A})-\mathrm{O}(22 \mathrm{~A})$ & $1.506(11)$ & $\mathrm{P}(2 \mathrm{~A})-\mathrm{O}(23 \mathrm{~A})$ & $1.561(10)$ \\
\hline $\mathrm{P}(3 \mathrm{~A})-\mathrm{O}(23 \mathrm{~A})$ & $1.655(10)$ & $\mathrm{P}(3 \mathrm{~A})-\mathrm{O}(31 \mathrm{~A})$ & $1.486(8)$ \\
\hline $\mathrm{P}(3 \mathrm{~A})-\mathrm{O}(32 \mathrm{~A})$ & $1.538(10)$ & $\mathrm{P}(3 \mathrm{~A})-\mathrm{O}(33 \mathrm{~A})$ & $1.453(9)$ \\
\hline $\mathrm{Cu}(2)-\mathrm{O}(11 \mathrm{~B})$ & $2.273(9)$ & $\mathrm{Cu}(2)-\mathrm{O}(21 \mathrm{~B})$ & $1.977(9)$ \\
\hline $\mathrm{Cu}(2)-\mathrm{O}(31 \mathrm{~B})$ & $1.919(8)$ & $\mathrm{Cu}(2)-\mathrm{O}(33 \mathrm{~A})$ & $2.273(9)$ \\
\hline $\mathrm{Cu}(2)-\mathrm{N}(10 \mathrm{~B})$ & $2.050(12)$ & $\mathrm{Cu}(2)-\mathrm{N}(20 \mathrm{~B})$ & $1.993(11)$ \\
\hline $\mathrm{P}(1 \mathrm{~B})-\mathrm{O}\left(5^{\prime} \mathrm{B}\right)$ & $1.586(8)$ & $\mathrm{P}(1 \mathrm{~B})-\mathrm{O}(11 \mathrm{~B})$ & $1.454(9)$ \\
\hline $\mathrm{P}(1 \mathrm{~B})-\mathrm{O}(12 \mathrm{~B})$ & $1.490(8)$ & $\mathrm{P}(1 \mathrm{~B})-\mathrm{O}(13 \mathrm{~B})$ & $1.601(8)$ \\
\hline $\mathrm{P}(2 \mathrm{~B})-\mathrm{O}(13 \mathrm{~B})$ & $1.596(9)$ & $\mathrm{P}(2 \mathrm{~B})-\mathrm{O}(21 \mathrm{~B})$ & $1.470(10)$ \\
\hline $\mathrm{P}(2 \mathrm{~B})-\mathrm{O}(22 \mathrm{~B})$ & $1.468(11)$ & $\mathrm{P}(2 \mathrm{~B})-\mathrm{O}(23 \mathrm{~B})$ & $1.611(10)$ \\
\hline $\mathrm{P}(3 \mathrm{~B})-\mathrm{O}(23 \mathrm{~B})$ & $1.608(10)$ & $\mathrm{P}(3 \mathrm{~B})-\mathrm{O}(31 \mathrm{~B})$ & $1.504(9)$ \\
\hline $\mathrm{P}(3 \mathrm{~B})-\mathrm{O}(32 \mathrm{~B})$ & $1.535(10)$ & $\mathrm{P}(3 \mathrm{~B})-\mathrm{O}(33 \mathrm{~B})$ & $1.476(9)$ \\
\hline
\end{tabular}

Table IV. Selected bond lengths and angles in $\mathbf{2}$.

b) Bond angles $\left(^{\circ}\right)$

\begin{tabular}{lrlr}
\hline $\mathrm{N}(10 \mathrm{~A})-\mathrm{Cu}(1)-\mathrm{O}(21 \mathrm{~A})$ & $173.5(4)$ & $\mathrm{N}(20 \mathrm{~A})-\mathrm{Cu}(1)-\mathrm{O}(31 \mathrm{~A})$ & $171.0(4)$ \\
$\mathrm{O}(33 \mathrm{~B})-\mathrm{Cu}(1)-\mathrm{O}(21 \mathrm{~A})$ & $89.4(4)$ & $\mathrm{O}(33 \mathrm{~B})-\mathrm{Cu}(1)-\mathrm{O}(31 \mathrm{~A})$ & $95.9(3)$ \\
$\mathrm{O}(33 \mathrm{~B})-\mathrm{Cu}(1)-\mathrm{N}(10 \mathrm{~A})$ & $88.6(4)$ & $\mathrm{O}(33 \mathrm{~B})-\mathrm{Cu}(1)-\mathrm{N}(20 \mathrm{~A})$ & $90.8(4)$ \\
$\mathrm{P}(1 \mathrm{~A})-\mathrm{O}(13 \mathrm{~A})-\mathrm{P}(2 \mathrm{~A})$ & $131.0(6)$ & $\mathrm{P}(2 \mathrm{~A})-\mathrm{O}(23 \mathrm{~A})-\mathrm{P}(3 \mathrm{~A})$ & $130.0(5)$ \\
$\mathrm{N}(10 \mathrm{~B})-\mathrm{Cu}(2)-\mathrm{O}(21 \mathrm{~B})$ & $173.8(4)$ & $\mathrm{N}(20 \mathrm{~B})-\mathrm{Cu}(2)-\mathrm{O}(31 \mathrm{~B})$ & $168.7(4)$ \\
$\mathrm{O}(33 \mathrm{~A})-\mathrm{Cu}(2)-\mathrm{O}(21 \mathrm{~B})$ & $86.6(4)$ & $\mathrm{O}(33 \mathrm{~A})-\mathrm{Cu}(2)-\mathrm{O}(31 \mathrm{~B})$ & $94.7(3)$ \\
$\mathrm{O}(33 \mathrm{~A})-\mathrm{Cu}(2)-\mathrm{N}(10 \mathrm{~B})$ & $91.7(4)$ & $\mathrm{O}(33 \mathrm{~A})-\mathrm{Cu}(2)-\mathrm{N}(20 \mathrm{~B})$ & $92.4(4)$ \\
$\mathrm{P}(1 \mathrm{~B})-\mathrm{O}(13 \mathrm{~B})-\mathrm{P}(2 \mathrm{~B})$ & $129.8(5)$ & $\mathrm{P}(2 \mathrm{~B})-\mathrm{O}(23 \mathrm{~B})-\mathrm{P}(3 \mathrm{~B})$ & $130.8(6)$ \\
\hline
\end{tabular}

\section{Discussion}

Perspective drawings of the structures of the molecule of $\mathbf{2}$ and the cation of $\mathbf{3}$ are provided in Figs 1 and 2 . The structure of $\mathbf{2}$ is well determined and allows a more detailed discussion of the bonding geometry than was possible for the analogous complex $\left[\mathrm{Zn}\left(\mathrm{H}_{2} \mathrm{ATP}\right)(\text { bipy })\right]_{2} \cdot 4 \mathrm{H}_{2} \mathrm{O}$, for which $R=$ 0.098 for 1617 reflections [9]. The limited quality of the analysis of 3 prevents such an evaluation and in this case the discussion will concentrate on coordinational and conformational aspects of the structure.

Both copper atoms in $\mathbf{2}$ display a strongly distorted $[4+2]$-octahedral coordination with tridentate binding of the metal by individual ATP molecules. Equatorial ligands are provided by the $\mathrm{N}$ atoms of the phenanthrolines and by one $\beta$ - and one $\gamma$-phosphate $O$ atom (respectively $\mathrm{O} 21$ and 031) of an ATP molecule. The following bond distances are observed: $\mathrm{Cu}-\mathrm{O}_{\beta}$ 1.942(9), 1.977(9); $\mathrm{Cu}-\mathrm{O}_{\gamma}, 1.925(8), \quad 1.919(8) ; \quad\left(\mathrm{O}_{\beta}^{-}\right) \mathrm{Cu}-\mathrm{N} \quad 1.989(10)$, $2.050(12) ;\left(\mathrm{O}_{\gamma^{-}}\right) \mathrm{Cu}-\mathrm{N} 2.013(10), 1.993(11) \AA$. The coordination sphere is completed by an $\alpha$-phosphate $\mathrm{O}$ atom of the same ATP and a $\gamma$-phosphate $\mathrm{O}$ atom (respectively 011 and 033) of the second ATP moiety, thus yielding a dimeric structure with a central eight-membered ring, as typically observed for ternary metal complexes of nucleoside monophosphates $[2,3]$. The axial $\mathrm{Cu}-\mathrm{O}_{\alpha}$ interactions are very weak: $2.878(9)$ and $2.730(8) \AA$. It is of interest that these $\mathrm{O}$ atoms are also involved in strong intermolecular $\mathrm{O} \cdots \mathrm{H}-\mathrm{N}(6)$ hydrogen bonds of respective lengths 2.68 and $2.81 \AA$. In contrast, the axial $\mathrm{Cu}-\mathrm{O}_{\gamma}$ distances of $2.284(8)$ and 2.273(9) $\AA$ fall within the typical range for such interactions.

A dimeric structure is also observed for 3 , which displays bidentate equatorial coordination of the two central ring $\mathrm{Cu}$ atoms, $\mathrm{Cu}(2)$ and $\mathrm{Cu}(3)$, by an $\alpha$ - and a $\beta$-phosphate $\mathrm{O}$ atom (respectively $\mathrm{O} 11$ and O21) of an ADP molecule. In this case, however. the coordination of the copper atoms is $[4+1]$. square pyramidal with the metal shifted slightly from the plane of the four equatorial ligands towards the fifth axial ligand, which is provided by a $\beta$-phosphate $\mathrm{O}$ atom $\mathrm{O}(23)$, of the second ADP molecule. The axial $\mathrm{Cu}-\mathrm{O}_{\beta}$ interaction is significantly weaker than the equatorial $\mathrm{Cu}-\mathrm{O}$ interactions. An average value of $2.29 \AA$ is obtained for this bond 


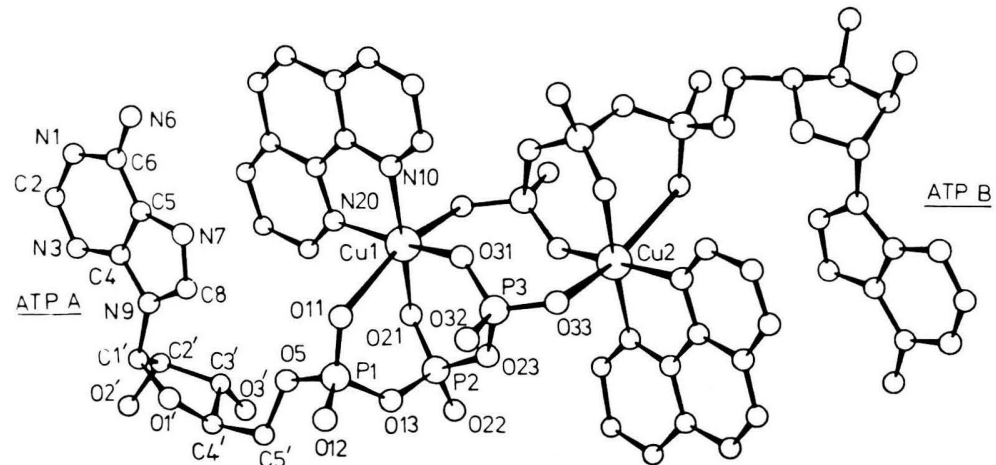

Fig. 1. Structure of $\left[\mathrm{Cu}\left(\mathrm{H}_{2} \mathrm{ATP}\right)(\text { phen })\right]_{2}(\mathbf{2})$. as compared to $1.92 \AA$ for the latter bonds. Perhaps the most surprising aspect of the structure of $\mathbf{3}$ is the monodentate coordination of two further copper atoms $\mathrm{Cu}(1)$ and $\mathrm{Cu}(4)$ by the $\beta$-phosphate $\mathrm{O}$ atoms $\mathrm{O}(22)$. These copper atoms also exhibit square pyramidal geometries with the equatorial substituents provided by two phenanthroline $\mathrm{N}$ atoms and a nitrato $\mathrm{O}$ atom, in addition to the $\beta$-phosphate $\mathrm{O}$ atom. The axial ligand is a water oxygen in each case. Inspection of Fig. 1 indicates that the free terminal $\gamma$-phosphate $\mathbf{O}$ atom in $\mathbf{2}$ is not capable of binding a further metal ion for steric reasons. In 3, however, the atom $\mathrm{O}(22)$ of the terminal $\beta$-phosphate are directed away from the chelate sixmembered ring and the central eight-membered ring and are, therefore, sterically available for further metal binding.

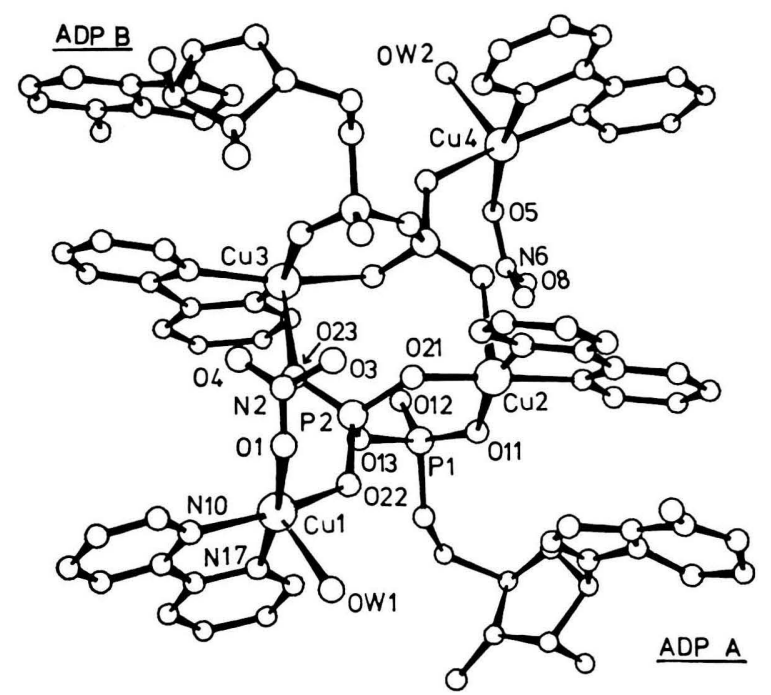

Fig. 2. Structure of

$\left[\mathrm{Cu}_{4}(\mathrm{HADP})_{2}(\text { bipy })_{4}\left(\mathrm{H}_{2} \mathrm{O}\right)_{2}\left(\mathrm{NO}_{3}\right)_{2}\right]^{2+}(\mathbf{3})$.
Intra- and intermolecular stacking of the heterocyclic bases is observed for both 2 and 3 . The intramolecular interactions between the adenine bases and the second chelating ligands are clearly of importance for the stability of such complexes. The geometric nature of this stacking is depicted in Figs 3 and 4, which show projections perpendicular to the purine base planes. Closest contacts are observed for $\mathrm{C}(8)$ of ATP molecule A [3.32 $\AA], \mathrm{C}(5)$
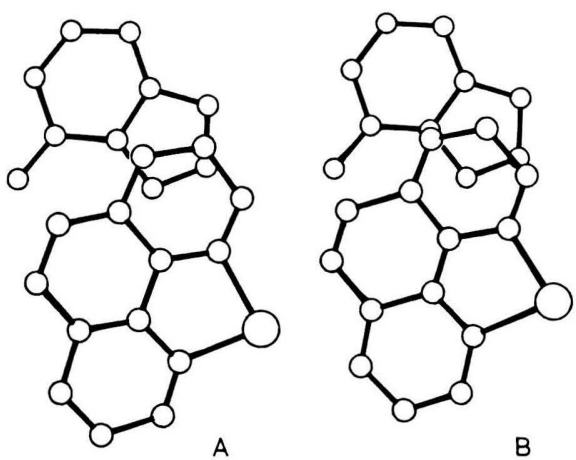

Fig. 3. Intramolecular stacking of the adenine and phenanthroline systems in $\mathbf{2}$. Projections are perpen. dicular to the adenine planes.
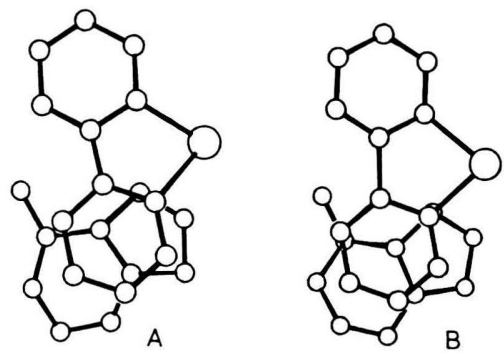

Fig. 4. Intramolecular stacking of the adenine and bipyridyl systems in 3. Projections are perpendicular to the adenine planes. 
of ATP molecule B [3.48 $\AA$ ], C(4) of ADP molecule A $[3.35 \AA]$ and $\mathrm{C}(6)$ of ADP molecule B [3.21 $\AA$ ]. The base planes are not exactly parallel to one another. Dihedral angles of $6.7,5.8,6.8$ and $4.7^{\circ}$ are observed. Intramolecular stacking of the adenine systems with neighbouring bipyridyl moieties in 3 is only achieved at the energetic expense of the adoption of unusually low absolute values of the torsion angle $\varnothing=$ $\mathrm{C}\left(4^{\prime}\right)-\mathrm{C}\left(5^{\prime}\right)-\mathrm{O}\left(5^{\prime}\right)-\mathrm{P}(1)$ (Table $\left.\mathrm{V}\right)$. A value close to the ideal value of $180^{\circ}$ is observed for this angle in $5^{\prime}$-monophosphates [13]. Lower values for $\varnothing$ have, however, been found in the crystal structures of salts of nucleoside di- and triphosphates, e.g. $144.6^{\circ}$ in KADP [14] and $-138.4^{\circ}$ and $-142.4^{\circ}$ for the two independent molecules of $\mathrm{Na}_{2}$ ATP [15]. Basephosphate interactions fold the phosphate chain towards the base and lead to $\varnothing$ values deviating from the ideal trans value in these nucleotides. An even greater degree of folding is necessary in $\mathbf{3}$ to enable base stacking. In this case $\varnothing$ values of 98 and $-102^{\circ}$ are observed. ADP molecule B also adopts the unfavourable eclipsed conformation for the torsion angle $\mathrm{C}\left(5^{\prime}\right)-\mathrm{O}\left(5^{\prime}\right)-\mathrm{P}(1)-\mathrm{O}(12)$. The ATP molecules in $\mathbf{2}$ display much larger absolute values of $\varnothing$ of -147.1 and 170.0, indicating that the geometrical prerequisites for base stacking are more favourable in this case. It is, however, interesting to note, in this context, that the degree of stacking overlap for the ADP adenines in 3 is greater than that for the ATP adenines in 2 (Figs 3 and 4). In contrast to the conformation at $\mathrm{C}\left(5^{\prime}\right)-\mathrm{O}\left(5^{\prime}\right)$ values of $\chi_{\mathrm{CN}}$ and $\psi_{\mathrm{OC}}$ in 3 (Table $\mathrm{V}$ ) lie in the typical ranges for non-complexed purine nucleotides. The conformation at the glycosidic bond $\mathrm{N}(9)-\mathrm{C}(1)$ is anti for both independent nucleotides of $\mathbf{2}$ and $\mathbf{3}$. Likewise the conformation at $\mathrm{C}\left(4^{\prime}\right)-\mathrm{C}\left(5^{\prime}\right)$ is the common gauche. The bipyridyl ligands of $\mathrm{Cu}(1)$ and $\mathrm{Cu}(4)$ in 3 are only involved in intermolecular stacking (with one another).

The structures of $\mathbf{2}$ and $\mathbf{3}$ are essentially in agreement with the models proposed by Sigel on the basis of his solution studies [7]. Chelation of the copper ions by bipyridyl or phenanthroline prevents the interaction of the metal ion with the purine nitrogen $\mathrm{N}(7)$, as commonly observed in binary complexes of 5 -monophosphates $[2,3]$. This appears to be an essential step in the hydrolysis mechanism for nucleoside polyphosphates [16]. These ternary complexes are, therefore, potential models for the mechanism of ATP and ADP transport in biological media. The structure of $\left[\mathrm{Cu}\left(\mathrm{H}_{2} \mathrm{ATP}\right)(\text { phen })\right]_{2}$ also lends valuable support to the mechanism which has been proposed for the enzymatic phosphate transfer from ATP [17, 18]. 1,10-Phenanthroline may be regarded as a simple model for an enzyme which binds $\mathrm{M}(\mathrm{ATP})^{2-}$ more strongly than $\mathrm{M}^{2+}$ or $\mathrm{ATP}^{4-}$ e.g. the system arginine kinase/Mn(ATP $)^{2-}[19]$. Then the present results, taken together with the

Table V. Conformations of the nucleoside polyphosphates in $\mathbf{2}$ and $\mathbf{3}$.

\begin{tabular}{|c|c|c|c|c|}
\hline & $\stackrel{2}{\text { Molecule A }}$ & Molecule B & $\begin{array}{l}\mathbf{3} \\
\text { Molecule A }\end{array}$ & Molecule B \\
\hline Glycosidic torsion angle & anti & anti & anti & anti \\
\hline$\chi_{\mathrm{CN}}=\mathrm{O}\left(\mathrm{l}^{\prime}\right)-\mathrm{C}\left(\mathrm{l}^{\prime}\right)-\mathrm{N}(9)-\mathrm{C}(8)$ & $32.3^{\circ}$ & $5.7^{\circ}$ & $47^{\circ}$ & $3^{\circ}$ \\
\hline Ribose conformation ${ }^{\mathrm{a}}$ & C $3^{\prime}$-endo & C $3{ }^{\prime}$-endo & & \\
\hline Conformation of $\mathrm{C}\left(5^{\prime}\right)-\mathrm{O}\left(5^{\prime}\right)$ & gauche ${ }^{+}$ & gauche $^{+}$ & gauche $\theta^{+}$ & gauche ${ }^{+}$ \\
\hline$\psi_{\mathrm{OC}}=\mathrm{O}\left(5^{\prime}\right)-\mathrm{C}\left(5^{\prime}\right)-\mathrm{C}\left(4^{\prime}\right)-\mathrm{C}\left(3^{\prime}\right)$ & $59.6^{\circ}$ & $50.1^{\circ}$ & $68^{\circ}$ & $72^{\circ}$ \\
\hline \multicolumn{5}{|c|}{ Torsion angles of the phosphate chain $\left({ }^{\circ}\right)$} \\
\hline$\varnothing=\mathrm{C}\left(4^{\prime}\right)-\mathrm{C}\left(5^{\prime}\right)-\mathrm{O}\left(5^{\prime}\right)-\mathrm{P}(1)$ & -147.1 & 170.0 & 98 & -102 \\
\hline $\mathrm{C}\left(5^{\prime}\right)-\mathrm{O}\left(5^{\prime}\right)-\mathrm{P}(1)-\mathrm{O}(11)$ & -172.6 & -169.1 & -98 & 123 \\
\hline $\mathrm{C}\left(5^{\prime}\right)-\mathrm{O}\left(5^{\prime}\right)-\mathrm{P}(1)-\mathrm{O}(12)$ & 57.0 & -37.2 & 43 & 0 \\
\hline $\mathrm{C}\left(5^{\prime}\right)-\mathrm{O}\left(5^{\prime}\right)-\mathrm{P}(1)-\mathrm{O}(13)$ & -55.4 & 71.9 & 153 & -128 \\
\hline $\mathrm{O}\left(5^{\prime}\right)-\mathrm{P}(1)-\mathrm{O}(13)-\mathrm{P}(2)$ & -71.2 & 84.6 & 120 & - 99 \\
\hline $\mathrm{O}(11)-\mathrm{P}(1)-\mathrm{O}(13)-\mathrm{P}(2)$ & 42.8 & -29.9 & 10 & 12 \\
\hline $\mathrm{P}(1)-\mathrm{O}(13)-\mathrm{P}(2)-\mathrm{O}(21)$ & 9.1 & -16.2 & 18 & -26 \\
\hline $\mathrm{P}(1)-\mathrm{O}(13)-\mathrm{P}(2)-\mathrm{O}(23)$ & -108.7 & 99.3 & 137 & -148 \\
\hline $\mathrm{O}(13)-\mathrm{P}(2)-\mathrm{O}(23)-\mathrm{P}(3)$ & 76.6 & -75.2 & & \\
\hline $\mathrm{P}(2)-\mathrm{O}(23)-\mathrm{P}(3)-\mathrm{O}(31)$ & 29.3 & -32.4 & & \\
\hline $\mathrm{P}(2)-\mathrm{O}(23)-\mathrm{P}(3)-\mathrm{O}(33)$ & 155.8 & -157.4 & & \\
\hline Conformation of the phosphate chain & folded & folded & & \\
\hline$L_{-}(\mathrm{P}(1) \cdots \mathrm{P}(2) \cdots \mathrm{P}(3))^{1}$ & $93.5^{\circ}$ & $88.8^{\circ}$ & & \\
\hline
\end{tabular}

a The quality of the analysis does not allow an unequivocal determination of the ribose conformations for $\mathbf{3}$. 
structure of $\left[\mathrm{Zn}\left(\mathrm{H}_{2} \mathrm{ATP}\right)(\text { bipy })\right]_{2}[9]$, indicate that charge-transfer interactions could play a significant role in the relative stability of enzyme- $\mathrm{I}^{2+}-\mathrm{ATP}$ (and ADP) complexes. Furthermore, the phosphate binding to the metal ion in $\mathbf{2}$ is essentially bidentate, involving the $\beta$ - and $\gamma$-phosphate $\mathrm{O}$ atoms. However, the fact that an $\alpha$-phosphate $\mathrm{O}$ atom also makes a weak bond to the metal ion demonstrates that the geometrical prerequisites for a change from $\beta, \gamma-$ to $\alpha, \beta$-coordination are provided. Shortening of the $\mathrm{Cu}-\mathrm{O}_{\alpha}$ bond accompanied by a corroborative length- ening of the $\mathrm{Cu}-\mathrm{O}_{\gamma}$ bond will lead to the latter coordination and hence to a labile $\gamma$-phosphate group, which is then available for transfer.

A comparison of the present ternary copper complexes of ATP and ADP indicates that the geometrical conditions for intramolecular base stacking are more favourable in the ATP complex. However, an additional stabilisation of the ADP complex is provided by the monodentate binding of a further copper ion by each of the free terminal $\beta$-phosphate $\mathrm{O}$ atoms.
[1] Transition Metal Complexes of Purine Nucleotides, Part III. Part II : W. S. Sheldrick, Acta Crystallogr. B 37, 1820 (1981).

[2] V. Swaminathan and M. Sundarlingam, CRC Crit. Rev. Biochem. 6, 245 (1979).

[3] R. W. Gellert and R. Bau, Metal Ions in Biological Systems, edited by H. Sigel, Vol. 8, pp. 57-124, Marcel Dekker, New York, Basel 1979.

[4] R. D. Cornelius, P. A. Hart, and W. W. Cleland, Inorg. Chem. 16, 2799 (1977).

[5] T. M. Li. A. S. Mildvan, and R. L. Switzer, J. Biol. Chem. 253, 3918 (1978).

[6] M. Tetas and J. M. Lowenstein, Biochemistry 2, 350 (1963).

[7] C. F. Naumann and H. Sigel, J. Am. Chem. Soc. 96, 2750 (1974) ; P. R. Mitchell and H. Sigel, ibid. 100,1564 (1978).

[8] R. Cini and P. Oriolo, J. Inorg. Biochem. 14, 95 (1981).

[9] P. Orioli, R. Cini, D. Donati, and S. Mangani, Nature (London) 283, 691 (1980); J. Am. Chem. Soc. 103, 4446 (1981).
[10] W. S. Sheldrick, Angew. Chem. 93, 473 (1981).

[11] W. Saenger, Angew. Chem. 85̃, 680 (1973).

[12] Z. Shakked and D. Rabinovich, Proceedings of the Fourth European Crystallography Meeting, Abstract PI. 23, S. 142 (1977).

[13] M. Sundaralingam, Ann. NY Acad. Sci. 255, 3 (1975).

[14] P. Swaminathan and M. Sundaralingam, Acta Crystallogr. B 36, 2590 (1980).

[15] O. Kennard, N. W. Isaacs, W. D. S. Motherwell, J. C. Coppola, D. L. Wampler, A. C. Larson, and D. G. Watson, Proc. Roy. Soc. (London) A 325, 401 (1972).

[16] H. Sigel, D. H. Buisson, and B. Prijis, Bioinorg. Chem. 5, 1 (1975).

[17] H. Sigel and P. E. J. Amsler, J. Am. Chem. Soc. 98, 7390 (1976).

[18] D. Dunaway-Mariano, J. L. Benovic, W. W. Cleland, R. K. Gupta, and A. S. Mildvan, Biochemistry 18, 4347 (1979). 